\title{
COVID-19 in Hospitalized Patients on Chronic Peritoneal Dialysis: A Case Series
}

\author{
Mala Sachdeva ${ }^{a}$ Nupur N. Uppal ${ }^{a}$ Jamie S. Hirscha, b, c Jia H. Ng $^{a}$ \\ Deepa Malieckal $^{\mathrm{a}}$ Steven Fishbane ${ }^{\mathrm{a}}$ Kenar D. Jhaveri ${ }^{\mathrm{a}}$ the Northwell \\ COVID-19 Research Consortium and the Northwell Nephrology COVID-19 \\ Research Consortium
}

aDivision of Kidney Diseases and Hypertension, Department of Medicine, Donald and Barbara Zucker School of Medicine at Hofstra/Northwell, Great Neck, NY, USA; 'bnstitute of Health Innovations and Outcomes Research, Feinstein Institutes for Medical Research, Manhasset, NY, USA; 'Department of Information Services, Northwell Health, New Hyde Park, NY, USA

\section{Keywords}

Coronavirus disease 2019 - Nephrology · Peritoneal dialysis · End-stage kidney disease

\section{Abstract}

Background: The COVID-19 pandemic has affected the endstage kidney disease (ESKD) population, with high mortality rates reported among patients on hemodialysis. However, the degree to which it has affected the peritoneal dialysis (PD) population in the United States has not yet been elucidated. In this report, we describe the clinical characteristics, presentations, clinical course, and outcomes of ESKD patients on PD hospitalized with COVID-19. Methods: We describe the characteristics, presentation, and outcomes of adult ESKD patients on chronic PD hospitalized with COVID-19 in our 13 major hospitals in the NY health system using descriptive statistical analysis. Results: Of 419 hospitalized patients with ESKD, 11 were on chronic PD therapy (2.6\%). Among those 11,3 patients required mechanical ventilation, 2 of whom died. Of the entire cohort, 9 of the 11 patients (82\%) were discharged alive. While fever was a common presentation, more than half of our patients also pre- sented with diarrhea. Interestingly, 3 patients were diagnosed with culture-negative peritonitis during their hospitalization. Seven patients reported positive SARS-CoV-2 exposure from a member of their household. Conclusion: Hospitalized patients on PD with COVID-19 had a relatively mild course, and majority of them were discharged home.

(c) 2020 S. Karger AG, Basel

\section{Introduction}

In March 2020, New York City (NYC) became the epicenter of the coronavirus disease 2019 (COVID-19) pandemic caused by severe acute respiratory syndrome coronavirus 2 (SARS-CoV-2). To date, there have been 385,760 positive cases in New York state [1].

Patients with end-stage kidney disease (ESKD), including those on peritoneal dialysis (PD), have multiple comorbidities and a weakened immune system, making them more susceptible to infection than the general population [2]. During the COVID-19 pandemic, outpatient home dialysis center policies and procedures changed in order to practice better infection control and to decrease

\section{KARGER}

(C) 2020 S. Karger AG, Basel

karger@karger.com

www.karger.com/ajn
Mala Sachdeva

Division of Kidney Diseases and Hypertension

Donald and Barbara Zucker School of Medicine at Hofstra/Northwell, Northwell Health 100 Community Drive, Great Neck 11021 (USA)

msachdeva@northwell.edu 
Table 1. Demographic and clinical characteristics of hospitalized COVID-19 patients on chronic PD

\begin{tabular}{|c|c|}
\hline Variable & Total $(N=11)$ \\
\hline \multicolumn{2}{|l|}{ Age, years, $n(\%)$} \\
\hline$<50$ & $4(36)$ \\
\hline 50-59 & $3(27)$ \\
\hline $60-69$ & $3(27)$ \\
\hline $70-79$ & $1(9)$ \\
\hline Female, $n(\%)$ & $8(73)$ \\
\hline \multicolumn{2}{|l|}{ Race/ethnicity, $n(\%)$} \\
\hline Hispanic & $1(9)$ \\
\hline Non-Hispanic black & $5(45)$ \\
\hline Non-Hispanic white & $1(9)$ \\
\hline Other & $3(27)$ \\
\hline Unknown & $1(9)$ \\
\hline \multicolumn{2}{|l|}{ Language, $n(\%)$} \\
\hline English & $11(100)$ \\
\hline \multicolumn{2}{|l|}{ Insurance, $n(\%)$} \\
\hline Commercial & $1(9)$ \\
\hline Medicaid & $2(18)$ \\
\hline Medicare & $8(72)$ \\
\hline Self-pay & 0 \\
\hline Tertiary hospital, $n(\%)$ & $10(91)$ \\
\hline \multicolumn{2}{|l|}{$\mathrm{BMI}, \mathrm{kg} / \mathrm{m}^{2}, n(\%)$} \\
\hline 18.5 to $<25.0$ & $3(27)$ \\
\hline 25.0 to $<30.0$ & $4(36)$ \\
\hline$\geq 30.0$ & $4(36)$ \\
\hline \multicolumn{2}{|l|}{ Tobacco status, $n(\%)$} \\
\hline Never & $9(82)$ \\
\hline Former smoker & $2(18)$ \\
\hline Diabetes, $n(\%)$ & $5(45)$ \\
\hline Hypertension, $n(\%)$ & $10(91)$ \\
\hline CAD,$n(\%)$ & $1(9)$ \\
\hline Heart failure, $n(\%)$ & 0 \\
\hline PVD, $n(\%)$ & 0 \\
\hline Asthma, $n(\%)$ & $1(9)$ \\
\hline COPD, $n(\%)$ & 0 \\
\hline Liver disease, $n(\%)$ & 0 \\
\hline Kidney transplantation, $n(\%)$ & $1(9)$ \\
\hline Cancer, $n(\%)$ & 0 \\
\hline
\end{tabular}

CAD, coronary artery disease; COPD, chronic obstructive pulmonary disease; PVD, peripheral vascular disease; PD, peritoneal dialysis.

community spread. This pandemic has affected the ESKD population, with high mortality rates reported among patients on hemodialysis (HD) [3]. However, the degree to which it has affected the PD population in the United States (US) has not yet been elucidated $[4,5]$. Herein, we describe the clinical characteristics, presentations, clinical course, and outcomes of ESKD patients on PD hospitalized with COVID-19.
Table 2. Clinical presentation, imaging, medical therapy, and outcomes of hospitalized COVID-19 patients on chronic PD

\section{Patient}

$\begin{array}{lllllllllll}1 & 2 & 3 & 4 & 5 & 6 & 7 & 8 & 9 & 10 & 11\end{array}$

\begin{tabular}{|c|c|c|c|c|c|c|c|c|c|c|}
\hline \multicolumn{11}{|l|}{ Clinical presentation } \\
\hline Diarrhea & -+ & - & + & + & - & + & + & + & - & - \\
\hline Shortness of breath & $-\quad-$ & + & - & + & + & - & - & + & - & + \\
\hline Cough & $-\quad-$ & - & + & + & + & + & - & - & - & + \\
\hline Myalgia & -+ & - & - & - & + & - & - & + & - & + \\
\hline Fever & + & - & + & + & + & + & - & - & + & + \\
\hline Vertigo & +- & - & - & - & - & - & - & - & - & - \\
\hline Vomiting & +- & - & + & - & - & - & - & - & - & - \\
\hline Abdominal pain & -+ & - & - & - & - & - & + & - & - & - \\
\hline Agitation & - & + & - & - & - & - & - & - & - & - \\
\hline Loss of taste or smell & $-\quad-$ & - & - & - & - & - & - & + & - & - \\
\hline Chills & $-\quad-$ & - & + & - & - & - & - & - & + & - \\
\hline Peritonitis & -+ & - & + & - & - & - & + & - & - & - \\
\hline \multicolumn{11}{|l|}{ Chest imaging findings } \\
\hline Bilateral opacities & ++ & + & - & + & + & + & - & + & + & + \\
\hline Unilateral pneumonia & - & - & + & - & - & - & - & - & - & - \\
\hline Clear lung fields & $-\quad-$ & - & - & - & - & - & + & - & - & - \\
\hline ESA therapy & ++ & - & - & - & - & - & + & + & + & - \\
\hline \multicolumn{11}{|l|}{ COVID-19 therapy } \\
\hline Hydroxychloroquine & +- & + & + & - & + & + & - & - & - & + \\
\hline Azithromycin & -+ & + & + & - & - & - & - & - & - & + \\
\hline Tocilizumab & +- & + & - & - & - & - & - & - & - & + \\
\hline Methylprednisolone & $-\quad-$ & + & - & - & + & + & - & - & - & - \\
\hline Anakinra & - - & - & - & - & - & + & - & - & - & - \\
\hline Convalescent plasma & - & - & - & - & - & - & - & - & - & - \\
\hline \multicolumn{11}{|l|}{ Hospital course } \\
\hline Vasopressor requirement & - & + & - & - & + & - & - & - & - & - \\
\hline Mechanical ventilation & - & + & - & - & + & - & - & - & - & + \\
\hline Intensive care unit stay & -- & + & - & - & + & - & - & - & - & + \\
\hline \multicolumn{11}{|l|}{ Outcomes } \\
\hline Death & - & + & - & - & + & - & - & - & - & - \\
\hline Discharged home & ++ & - & + & + & - & + & + & + & + & + \\
\hline Re-admission & - & - & - & - & - & - & - & + & - & - \\
\hline
\end{tabular}

ESA, erythropoiesis-stimulating agent; COVID-19, coronavirus disease 2019; PD, peritoneal dialysis.

\section{Methods}

This is a case series of patients with ESKD on chronic PD hospitalized with COVID-19 in 13 hospitals of a large health system in the New York City area. Data were obtained from the enterprise inpatient electronic health record Sunrise Clinical Manager (Allscripts, Chicago, IL). All adult patients (age $\geq 18$ years) with prevalent ESKD on PD who tested positive by PCR testing of a nasopharyngeal sample for COVID-19 from March 1, 2020, to April 27,2020 , were eligible. The patients were followed up through June 15,2020 . For patients who had multiple qualifying hospital admissions, we included only the first hospitalization. 
Table 3. Baseline laboratory parameters of hospitalized COVID-19 patients on chronic PD

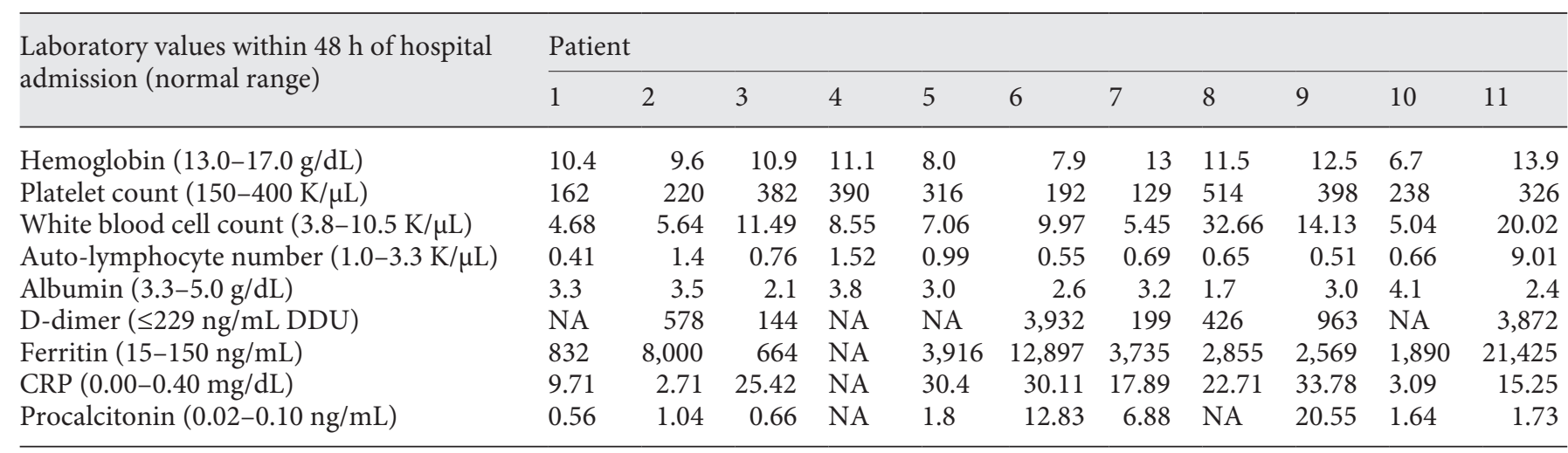

NA, not available; PD, peritoneal dialysis; CRP, C-reactive protein; COVID-19, coronavirus disease 2019.

For hospitalized patients, we identified patients with ESKD on PD through the following mechanisms: (1) presence of providerentered discrete comorbidity of ESKD (International Classification of Diseases, Tenth Revision [ICD-10] code N18.6); (2) independent adjudication of the ESKD diagnosis and PD status through manual chart review of hospital admission and nephrology consultation notes to ensure patients had prevalent ESKD (J.S.H.); and (3) presence of dialysis orders specifically for the PD procedure.

We collected data on patient demographics, baseline history of comorbid conditions, medications, dialysis-specific data elements, and details of the hospital admissions. Comorbid conditions and home medications were determined from provider-entered past medical history and admission medication reconciliation. Clinical presentation, imaging findings, episode of peritonitis, modality of $\mathrm{PD}, \mathrm{PD}$ orders, and ventilatory settings were collected using manual chart review (M.S., D.M., and N.N.U.). We collected details during hospital admission such as intensive care unit (ICU) stay, need for mechanical ventilation, vasopressor support, hospital length of stay, death, discharge, and baseline laboratory test results within $48 \mathrm{~h}$ of hospital admission. Due to the COVID-19 pandemic, many additional ICUs were created in nontraditional hospital areas and units. Hence, ICU stay was defined as either one of the following: need for invasive mechanical ventilation, need for vasopressor or inotrope support, under the care of an ICU service, or in a known ICU location. As the overall number of patients receiving PD was small, only descriptive statistical analysis was performed. The Institutional Review Board of Northwell Health approved the study protocol before the commencement of the study.

\section{Results}

We identified 11 patients with ESKD on PD hospitalized with COVID-19 (out of a total of 419 hospitalized patients with ESKD [2.6\%]). The median age was 54 years, with $4(36 \%)$ patients aged less than 40, 3 (27\%) aged 5059 , and $3(27 \%)$ aged $60-69$ years. The majority of those admitted were female (8 of 11 [73\%]) and 5 (45\%) were non-Hispanic black. The patients were on an average of 8 home medications, 2 were on angiotensin-converting enzyme inhibitors, and 1 was on immunosuppressive medications (prednisone and tacrolimus). Five of the 11 patients received erythropoietin-stimulating agents during the hospitalization.

Ten patients had hypertension, 5 had diabetes mellitus, 2 had systemic lupus erythematosus, 1 had coronary artery disease, 1 had asthma, and 1 had a history of a prior failed kidney transplant. None of the patients had any underlying malignancy. Four (36\%) patients were overweight, and another $4(36 \%)$ were obese. Nine reported no smoking history, and 2 were former smokers. Table 1 summarizes the baseline demographics and characteristics of these patients.

The most commonly reported presenting symptoms were fever (present in 7 of 11 [64\%]) and diarrhea (6 of 11 [55\%]), followed by shortness of breath (5 of 11 [45\%]), cough (5 of $11[45 \%]$ ), and myalgias (4 of 11 [36\%]). Less commonly reported symptoms included vomiting in 2 (18\%), abdominal pain in $2(18 \%)$, agitation in $1(9 \%)$, vertigo in $1(9 \%)$, and loss of taste or smell in $1(9 \%)$. Seven patients reported positive SARS-CoV-2 exposure from a member of their household.

Initial chest radiographs showed bilateral opacities in the majority, 9 of 11 patients (82\%). One patient had unilobar pneumonia, and another had no acute findings on chest radiograph. Table 2 summarizes the clinical presentation, imaging findings, and outcomes of these patients. Table 3 and online suppl. Table 1 (for all online suppl. material, see www.karger.com/doi/10.1159/000510259) summarize the pertinent laboratory data within the 48 
hours of admission for these patients and the inpatient orders for PD.

The hospital course was complicated by ICU stay in 3 of 11 patients (27\%), all of whom required mechanical ventilation. Among the patients ventilated and who were on $\mathrm{PD}$, one had a decrease in the plateau pressure from 36 to $29 \mathrm{~cm} \mathrm{H}_{2} \mathrm{O}$ and another had no significant change in plateau pressure, which remained at $20 \mathrm{~cm} \mathrm{H}_{2} \mathrm{O}$ despite being on $\mathrm{PD}$. Seven patients received continuous ambulatory PD (CAPD), and 4 patients were on automated PD during the hospitalization. One patient in the ICU received HD temporarily for one day due to severe hyperkalemia, with subsequent transition back to CAPD.

Three patients were diagnosed with culture-negative peritonitis during hospitalization. None of the 3 patients with peritonitis died. Online suppl. Table 2 shows the cell counts of the patients treated for peritonitis and their antibiotic course and duration.

The length of hospital stay ranged from 2 to 23 days, with the average length of stay being 9 days. Two of the $11(18 \%)$ patients expired, both of whom required mechanical ventilation and died within 4 days of admission (Table 2). Both patients continued on CAPD until the day of expiration. Nine patients (82\%) were discharged home, and one was readmitted for worsening pneumonia.

\section{Discussion}

In this report, we describe the characteristics, presentation, and outcomes of ESKD patients on chronic PD hospitalized with COVID-19. Of 419 hospitalized patients with ESKD, 11 were on chronic PD therapy (2.6\%). Among those 11, three patients required mechanical ventilation, two of whom died. Of the entire cohort, 9 of the 11 patients $(82 \%)$ were discharged alive. All but one patient were continued on PD without any significant electrolyte or volume-related concerns, and two of the patients on ventilator had either no change or improvement in their plateau pressures, making PD a viable option for those with COVID-19 infection and on mechanical ventilation.

Like all patients with ESKD, those on PD carry an increased risk of infection due to their immunosuppressed state and comorbid conditions. While some established risk factors for severe illness with COVID-19 such as advanced age, diabetes mellitus, and hypertension were present in our cohort, others such as heart disease, obesity, and cancer were less common or absent entirely [6]. Despite the risk factors, they seemed to do fairly well.
Data for hospitalized patients on HD are slowly emerging, yet there are no reports of the burden of illness of COVID-19 in the PD population. Most studies from Europe, the US, and China show a high mortality rate for HD patients $[3-5,7]$. In one single center study from the US, only 2 of 59 hospitalized patients with ESKD and COVID-19 were on PD [3]. We did not observe a large number of patients hospitalized with this illness possibly owing to the ability of PD patients to perform dialysis at home and socially distance from others as opposed to incenter HD patients, PD patients being highly trained toward proper hygiene, and new policies and procedures that were put in place by outpatient PD units in an effort to decrease risk. In our population of admitted patients on PD, most did not require mechanical ventilation and were discharged home. Despite that, given our small sample size, definitive conclusions regarding mortality in hospitalized patients on PD with COVID-19 are not possible.

Commonly reported symptoms associated with COVID-19 include fever, cough, and shortness of breath [8]. While fever was a common presentation, more than half of our patients also presented with diarrhea. One patient presented with diarrhea, abdominal pain, and myalgias, and another presented with only diarrhea and abdominal pain. Neither of these patients had the typical cough or shortness of breath. Our findings suggest the importance of assessing atypical symptoms of COVID-19, especially gastrointestinal symptoms in the $\mathrm{PD}$ population, and taking appropriate infection control precautions and diagnostic measures when these atypical symptoms are identified.

Interestingly, 3 patients were diagnosed with culturenegative peritonitis during their hospitalization. None of these patients died. Owing to the temporal relationship of the COVID-19 illness and peritonitis, it is possible that SARS-CoV-2 might have been responsible for the peritonitis. Whether this is a direct effect of the virus, hematogenous spread, touch contamination, the effect of inflammatory mediators, superimposed bacterial translocation due to diarrhea, or another issue remains uncertain, although Vischini et al. [9] previously reported peritoneal dialysate being positive for SARS-CoV-2 in a COVID-19 patient.

Limitations of this case series include the small sample size and the observational nature of the data collection from the electronic medical record. The study was unable to obtain prevalence data as several different outpatient units admit to our enterprise hospitals and our electronic medical record system was not able to capture 
that. However, these data come from a diverse population of over 11,000 patients hospitalized at 13 hospitals in the New York area, the epicenter of the COVID-19 outbreak.

\section{Conclusion}

In conclusion, we describe a series of patients with ESKD on chronic PD hospitalized with COVID-19 disease during the peak of the pandemic. We found that most patients had a relatively mild course and were discharged home. Further studies are needed to understand the absolute risk of infection in patients on PD and the spectrum of the burden of illness including presentation, complications of illness, and outcomes.

\section{Acknowledgements}

The authors would like to acknowledge and thank our nursing staff that include Myung Cho, Virginia Crosby, Jennifer Koczko, Joanne Muccio, Jennie Wilson, and all other teammates for their hard work and commitment to our home dialysis programs.

\section{Statement of Ethics}

This study was approved by the Northwell IRB (COVID IRB \# 529).

\section{Conflict of Interest Statement}

K.D.J. is a consultant for Astex Pharmaceuticals and Natera.

\section{Funding Sources}

No funding was needed for this study.

\section{Author Contributions}

M.S., N.N.U., and D.M. made substantial contributions to the study concept and design. M.S. was in charge of the manuscript draft. J.S.H. was responsible for data extraction. J.H.N., S.F., and K.D.J. helped in supervising and editing of the manuscript. M.S. and N.N.U. participated in drafting the manuscript and revising it on the basis of reviewers' comments. J.N. and J.S.H. made substantial contributions to data acquisition, analysis, and interpretation. All Northwell consortium authors (listed in online suppl. material) were involved in editing of the final manuscript.

\section{References}

1 Workbook. NYS-COVID19-Tracker [internet]. 2020 https://covid19tracker.health.ny. gov/views/NYS-COVID19-Tracker/NYSDOHCOVID-19Tracker-Map?\%3Aembed = yes\&\%3Atoolbar $=$ no\&\%3Atabs $=\mathrm{n}$.

2 Kato S, Chmielewski M, Honda H, PecoitsFilho R, Matsuo S, Yuzawa Y, et al. Aspects of immune dysfunction in end-stage renal disease. Clin J Am Soc Nephrol 2008;3(5):152633.

3 Valeri AM, Robbins-Juarez SY, Stevens JS, Ahn W, Rao MK, Radhakrishnan J, et al. Presentation and outcomes of patients with ESKD and COVID-19. J Am Soc Nephrol. 2020;31(7):1409-15. https://jasn.asnjournals. org/content/early/2020/05/28/ASN. 2020040470.
4 Fisher M, Yunes M, Mokrzycki MH, Golestaneh L, Alahiri E, Coco M. Chronic hemodialysis patients hospitalized with COVID-19: short-term outcomes in Bronx, New York. Kidney360. 2020.

5 Alberici F, Delbarba E, Manenti C, Econimo L, Valerio F, Pola A, et al. A report from the Brescia Renal COVID Task Force on the clinical characteristics and short-term outcome of hemodialysis patients with SARS-CoV-2 infection. Kidney Int. 2020. http://www.sciencedirect.com/science/article/pii/ S0085253820305081.

6 Richardson S, Hirsch JS, Narasimhan M, Crawford JM, McGinn T, Davidson KW, et al. Presenting characteristics, comorbidities, and outcomes among 5700 patients hospitalized with COVID-19 in the New York city area. JAMA. 2020
7 Xiong F, Tang H, Liu L, Tu C, Tian J-B, Lei $\mathrm{C}-\mathrm{T}$, et al. Clinical characteristics of and medical interventions for COVID-19 in hemodialysis patients in wuhan, China. J Am Soc Nephrol. 2020.

8 Guan WJ, Ni ZY, Hu Y, Liang WH, Ou CQ, $\mathrm{He} \mathrm{JX}$, et al. Clinical characteristics of coronavirus disease 2019 in China. N Engl J Med. 2020;382(18):1708-20.

9 Vischini G, D’Alonzo S, Grandaliano G, D'Ascenzo FM. SARS-CoV-2 in the peritoneal waste in a patient treated with peritoneal dialysis. Kidney Int. 2020. 